\title{
Systemic oxidative stress and cognitive function in Parkinson's disease with different PWMH or DWMH lesions
}

\author{
Ta-Chih Chang ${ }^{1 \dagger}$, Yi-Cun Chen ${ }^{1+}$, Yu-Chi Huang ${ }^{1 *+} \mathbb{D}$, Wei-Che Lin $^{2^{*+}}$ and Cheng-Hsien Lu $^{3}$
}

\begin{abstract}
Background: Parkinson's disease (PD), frequently accompanied by cognitive impairments, is associated with systemic oxidative stress and abnormal structural changes on brain images. We aimed to identify the correlation between systemic oxidative stress and cognitive function in PD patients with different periventricular white matter hyperintensities (PWMH) and deep white matter hyperintensities (DWMH).

Methods: A total of 146 participants with idiopathic PD underwent brain MRI, which revealed PWMH and DWMH. The number of lesions were evaluated using the Fazekas criteria. Systemic oxidative stress was determined as early or late phase changes in leukocyte apoptosis and its subsets by flow cytometry. Cognitive functions, including attention, executive function, memory, language, and visual space, were assessed.

Results: For different DWMH, the leukocyte apoptosis and its subsets were significantly different.. However, there were no significant differences in oxidative stress biomarkers in PD patients with different PWMH. Attention and memory were significantly decreased in patients with more advanced DWMH injuries. Attention, memory, and language were significantly impaired in patients with worse PWMH lesions.

Conclusion: Significant oxidative stress biomarker alternations in PD patients with DWMH, but not PWMH, might be associated with white matter injury. Systemic inflammatory responses may contribute to deep white matter damage in PD. Further, more cognitive deficits were seen in PD patients with worse deep white matter lesions, especially in moderate to severe periventricular white matter injury.
\end{abstract}

Trial registration: Retrospective study.

Keywords: Oxidative stress, Cognitive function, Parkinson's disease, White matter injury

\footnotetext{
* Correspondence: hyuchi@gmail.com; u64lin@yahoo.com.tw

${ }^{\dagger} \mathrm{Ta}$-Chih Chang and Yi-Cun Chen contributed equally to this work and share the role of first author.

†Yu-Chi Huang and Wei-Che Lin contributed equally to this work and share the role of corresponding author.

'Department of Physical Medicine and Rehabilitation, Kaohsiung Chang

Gung Memorial Hospital and Chang Gung University College of Medicine,

Kaohsiung, Taiwan

${ }^{2}$ Department of Diagnostic Radiology, Kaohsiung Chang Gung Memorial

Hospital and Chang Gung University College of Medicine, Kaohsiung, Taiwan

Full list of author information is available at the end of the article
}

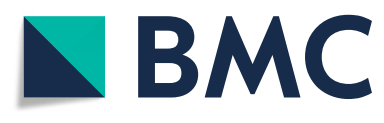

(c) The Author(s). 2021 Open Access This article is licensed under a Creative Commons Attribution 4.0 International License, which permits use, sharing, adaptation, distribution and reproduction in any medium or format, as long as you give appropriate credit to the original author(s) and the source, provide a link to the Creative Commons licence, and indicate if changes were made. The images or other third party material in this article are included in the article's Creative Commons licence, unless indicated otherwise in a credit line to the material. If material is not included in the article's Creative Commons licence and your intended use is not permitted by statutory regulation or exceeds the permitted use, you will need to obtain permission directly from the copyright holder. To view a copy of this licence, visit http://creativecommons.org/licenses/by/4.0/ The Creative Commons Public Domain Dedication waiver (http://creativecommons.org/publicdomain/zero/1.0/) applies to the data made available in this article, unless otherwise stated in a credit line to the data. 


\section{Background}

Parkinson's disease (PD), which is frequently accompanied by cognitive dysfunction, is one of the most common neurodegenerative disorders in the elderly add reference 1 [1]. In PD, loss of neurons occurs in the substantia nigra and in the nigrostriatal pathway to the striatum, along with dopamine depletion [2]. Dopamine depletion from the basal ganglia, leading to disruption of the connections between the thalamus and motor cortex, is related to motor symptoms in PD [3]. In addition to motor dysfunction in $\mathrm{PD}$, dopaminergic deficiency in interrupting the modulation of frontostriatal networks is associated with cognitive impairment [4]. Although the clear pathogenic mechanisms of neurodegeneration in PD are not yet understood, they may involve a cascade of events that include interactions between genetic and environmental factors [5], abnormalities in protein processing [6], immune regulation [7], mitochondrial dysfunction [8], inflammation [9], and oxidative stress [10].

Increased systemic oxidative is associated with neuroinflammation in PD and progression of disease through various pathways, including blood-brain barrier dysfunction, microglia activation, and infiltration of peripheral immune cells and circulating cytokines [7, 9, 11]. Increased oxidative stress has also been reported to be associated with cognitive impairment in PD. [12, 13] The infiltration of peripheral leucocyte apoptosis and peripheral leucocyte adhesion molecules was found to be one of the interactive pathways between systemic oxidative stress and neuroinflammation [14]. Furthermore, in patients with PD, leucocyte apoptosis was significantly high and was associated with striatal dopamine neuron loss, as determined by magnetic resonance imaging (MRI) [15]. This evidence suggests that peripheral immune cells, which reflect the level of systemic oxidative stress, might play important roles in the development of PD and cognitive impairment.

Brain white matter is vulnerable to oxidative stress due to its relatively low intrinsic antioxidative properties [16]. White matter hyperintensities (WMHs), also called leucoaraiosis or white matter lesions [17], are frequently observed on brain MRI scans in elderly individuals [18], and higher WMH burdens are associated with worse cognitive performance in the general population, patients with Alzheimer's disease, and patients with PD. [19-21] In addition, systemic inflammation was found to be associated with white matter damage in patients with PD [14], obstructive sleep apnoea [22], and several neurological diseases [23]. WMH was reported to be an important risk factor for PD with mild cognitive impairment (PD-MCI) [24], although the evidence was still conflicting and inconsistent [25]. WMH can be defined as periventricular white matter hyperintensities
(PWMH) and deep white matter hyperintensities (DWMH) [26] by visual rating scales used in clinical settings [27]. This distinction seems to reflect different functional, histopathological, and aetiological features $[28,29]$. Previous studies found that the elderly with PWMH, rather than DWMH, are associated with impaired cognitive function, especially in the executive function/processing speed domain, than the memory domain $[26,30]$. For PD-MCI patients, one study demonstrated that PWMH was associated with worse cognitive performance, specifically, executive impairment and visuospatial impairment [21].

The objectives of our study were (1) to investigate the associations of systemic oxidative stress biomarkers in PD patients with DWMH or PWMH and (2) to identify cognitive performance in PD patients with different classifications or levels of white matter injuries.

\section{Methods \\ Participants}

In this retrospective study, we recruited one hundred and forty-six idiopathic PD patients who participated in the previous trials conducted from January, 2011 to June, 2018. All these previous trials investigated the neuropsychological functions, oxidative stress, and brain MRI findings in the patients with PD. Idiopathic PD was diagnosed by experienced neurologists according to the United Kingdom Brain Bank criteria [31]. Patients with a history of other neurological or psychiatric disorders were excluded from the study. For each patient, the physical and neurological examinations and imaging assessments were all recorded in the $\mathrm{ON}$-medication state. The Chang Gung Medical Foundation Institutional Review Board approved the study and previous trials, and all of the participants or their guardians provided written informed consent, including this study and previous trials. This study was funded by NMRPG8J6022 (MOST 108-2314-B-182A-014-MY3), NMRPG8J0271 (MOST 108-2314-B-182A-017), and CMRPG8K0221.

\section{Disease severity and neuropsychological assessments}

For each patient with idiopathic PD, disease severity and functional condition were reported according to the results of the Unified Parkinson's Disease Rating Scale (UPDRS) [32], the modified Hoehn and Yahr Staging Scale (HYSS) [33], and the Schwab \& England Activities of Daily Living Scale (SE-ADLS) [34]. The sections of UPDRS consist of the following evaluations: mentation, behaviour, and mood and daily activities of speech, salivation, swallowing, handwriting, cutting food and handling utensils, dressing, hygiene, turning in bed, falling, freezing while walking, walking, tremor, sensory complaints, and motor capability. Higher scores represent more severe conditions in patients with PD. The 
modified HYSS was used to evaluate the severity of PD based on clinical presentations and functional ability from stages 1 to 5 (higher levels indicate higher severity of the disease). SE-ADLS was used to assess a person's daily function for PD, in which a score of $100 \%$ indicates complete independence and $0 \%$ indicates a bedridden status with vegetative functions. The Mini-Mental State Examination (MMSE) was used to assess cognitive function in patients with idiopathic PD. Additionally, the subtests from the Cognitive Ability Screening Instrument (CASI) [35] and the Wechsler Adult Intelligence Scale-III (WAIS-III) [36] were used to build a neuropsychological battery including five domains (attention, executive function, language, memory, and visuospatial function) [37]. A neuropsychological battery was performed by a clinical psychologist blinded to each patient's status. The attention function consisted of the digit span score from the WAIS-III and the attention and orientation scores from the CASI. Executive functions included the similarity, arithmetic, matrix reasoning, picture arrangement, and digit symbol coding scores in the WAIS-III and the abstract thinking and judgement score in the CASI. Speech and language functions were presented using the vocabulary and comprehension scores in the WAIS-III and the language score in the CASI. Memory function was assessed using the information score of the WAIS-III and the short- and long-term memory scores of the CASI, while the visuo-spatial function was assessed using the picture completion and block design scores from the WAIS-III and the drawing score from the CASI. Furthermore, these five cognitive domains were analysed with the average Z-score of all subtest scores.

\section{MRI acquisition}

MRI scans were acquired on a GE Signa $3 \mathrm{~T}$ whole-body MRI scanner (General Electric Healthcare, Milwaukee, WI, USA) using an eight-channel phase array head coil at the Kaohsiung Chang Gung Memorial Hospital in Taiwan. Whole brain 3-D T1 weighted images were collected for each participant using an inversion-recovery fluid-attenuated fast spoilt gradient-recalled echo pulse sequence with the following imaging parameters: repetition time (TR)/ echo time (TE)/inversion time (TI) $=9.5 / 3.9 / 450 \mathrm{~ms}$; flip angle $=20^{\circ}$; number of excitations $(\mathrm{NEX})=1$; field of view $(\mathrm{FOV})=240 \times 240 \mathrm{~mm}^{2}$; matrix size $=512 \times 512$; voxel size $=0.47 \times 0.47 \times 1.3 \mathrm{~mm}^{3}$; and slice number $=110$ axial slices (without interslice gaps). T2-weighted fast spin-echo sequence $(\mathrm{TR} / \mathrm{TE}=$ 4200/102 ms; echo train length $=18 ; \mathrm{NEX}=2$; FOV $=$ $240 \mathrm{~mm}^{2}$; slice thickness $=5 \mathrm{~mm}$; matrix size $=320 \times 256$ and 18 slices) and axial fluid-attenuated inversionrecovery sequence $($ FLAIR) $(\mathrm{TR} / \mathrm{TE} / \mathrm{TI}=8000 / 100 / 2000$ $\mathrm{ms} ; \mathrm{NEX}=1 ; \mathrm{FOV}=240 \mathrm{~mm}^{2}$; slice thickness $=5 \mathrm{~mm}$; matrix size $=320 \times 256$ and 18 slices) in the same imaging session.

We applied the Fazekas criteria [38-40] to define the gradings of the PWMH and DWMH scales on brain MRI scans. It described the severity of white matter lesions according to the size and confluence of lesions on the MRI scans; 0 indicated an absent lesion on the images. PWMH scale grading was from 0 to 3: 0 indicated an absent lesion; 1 indicated a "caps" or pencil-thin lining lesion; 2 indicated a smooth "halo" lesion; and 3 indicated an irregular periventricular signal lesion extending into the deep white matter. DWMH scale grading was from 0 to 3: 0 indicated an absent lesion; 1 indicated a punctate foci lesion; 2 indicated the beginning of a confluence lesion; and 3 indicated a large confluent area lesion.

PD patients with PWMH or DWMH were divided into three groups according to the severity of white matter lesions (grade 0: normal, grade 1: mild, and grade 2-3: moderate to severe white matter injuries).

\section{Blood sampling and assessment of systemic oxidative stress biomarkers}

All participants underwent blood sampling by venepuncture while the neuropsychological assessments and brain MRI evaluations were performed simultaneously. In this study, the percentage of peripheral leucocyte apoptosis was used to assess the oxidative stress. A detailed description of the assessment of leucocyte apoptosis has been presented in previous studies $[14,22]$.

The status of leucocyte apoptosis was assessed with APO 2.7-phycoerythrin (PE) (clone 2.7A6A3; Immunotech) to identify early and late apoptosis. Positive expression of APO 2.7-PE appears to be restricted to cells undergoing apoptosis. The presence of early apoptotic cells indicated that the apoptotic process was reversible in the early stage, but the presence of late apoptotic cells demonstrated that the cell membrane integrity was disrupted. Leucocytes and their subtypes were analysed according to the intensity of CD45 expression using flow cytometry. Results are expressed as a percentage of specific fluorescence-positive cells. Apoptotic cells were defined as those positive for APO 2.7. A database coordinator monitored all data collection and entry, both of which were checked for any inconsistencies.

\section{Statistical analysis}

Age, UPDRS, modified HYSS, SE-ADLS, PWMH grading, DWMH grading, and MMSE were analysed using frequency distribution. The Kruskal-Wallis $\mathrm{H}$ test was used for group comparisons of the blood test data in these three groups, and the Mann-Whitney U test was used for between-group comparisons. The scores of each neuropsychological assessment were first changed into $\mathrm{Z}$ 
scores and were further calculated based on five main cognitive categories: attention function, executive function, memory, speech and language, and visuospatial function, and the total of each score represented the ability to perform these psychological functions. Statistical significance was defined as a $p$ value $<0.05$. All statistical tests were performed using SPSS 19.0 (SPSS, Inc., Chicago, IL, USA).

\section{Results}

\section{Clinical characteristics in PD patients with white matter lesions}

The clinical characteristics of patients with idiopathic PD are presented in Table 1. A total of 146 patients with PD, including 64 males and 82 females, were enrolled in this study. The mean age of the participants was 63.68 years. The percentages of hypertension, diabetes mellitus, hypercholesterolemia and smoking were 40, 13, 21.2 and $2.7 \%$, respectively. The percentage of medications use that possible interfere with cognitive performance, which defined as Benzodiazepines (BZD), was 35.6\%. The mean total scores of UPDRS were 38.54, the mean stages in modified HYSS, the SE-ADLS, and MMSE were $1.94,82.96$, and 17.91, respectively. The mean grading of the PWMH and DWMH were 1.16 and 1.12, respectively.

Table 1 Clinical characteristics in PD

\begin{tabular}{ll}
\hline & PD ( $\boldsymbol{n = 1 4 6 )}$ \\
\hline Age (years, mean (SD)) & $63.68(10.69)$ \\
Sex (male, female) & $64 ; 82$ \\
HTN (number, percentage) & $54(40 \%)$ \\
DM (number, percentage) & $19(13 \%)$ \\
Hypercholesterolemia (number, percentage) & $31(21.2 \%)$ \\
Smoking (number, percentage) & $4(2.7 \%)$ \\
Medications (number, percentage) & $52(35.6 \%)$ \\
UPDRS I, mean (SD) & $3.41(2.47)$ \\
UPDRS II, mean (SD) & $10.21(7.81)$ \\
UPDRS III, mean (SD) & $24.91(16.37)$ \\
UPDRS total, mean (SD) & $38.54(24.92)$ \\
Modified Hoehn and Yahr scale, mean (SD) & $1.94(1.14)$ \\
Schwab and England ADL scale, mean (SD) & $82.96(17.91)$ \\
PWMH grading, mean (SD) & $1.16(0.59)$ \\
DWMH grading, mean (SD) & $1.12(0.66)$ \\
MMSE, mean (SD) & $24.42(4.92)$ \\
\hline AmOng the cinica
\end{tabular}

Among the clinical characteristics, age, UPDRS, Hoehn and Yahr scale, Schwab and England ADL scale, PWMH grading, DWMH grading and MMSE were analysed using frequency distribution

UPDRS The Unified Parkinson's Disease Rating Scale, ADL Activity of daily living, PWMH Periventricular white matter hyperintensity, DWMH Deep white matter hyperintensity, MMSE Mini-Mental State Examination, HTN Hypertension, DM Diabetes Mellitus; Medications, was defined as Benzodiazepines
Group comparisons of disease severity in PD patients with different DWMH or PWMH gradings

According to DWMH or PWMH gradings, 146 participants were divided into three groups (normal, mild, and moderate to severe) depending on white matter alternations on brain images. In the DWMH groups (Table 2), 24,80 , and 42 patients with PD were grouped based on the presence of normal, mild, and moderate to severe white matter lesions (grade $0,1,2-3$ ). In the PWMH groups (Table 3 ), there were 16,90 , and 40 patients with normal (grade 0 ), mild (grade 1 ), or moderate to severe (grade 2-3) white matter lesions. There was a significant difference in age among PD patients with different PWMH and DWMH gradings. In DWMH, there was a significant difference in hypertension between mild and moderate to severe white matter lesions. In PWMH, there was significant difference in medications use among different gradings. No significant differences were revealed in gender, total UPDRS, Modified HYSS, and SE-ADLS among the groups with different PWMH and DWMH gradings.

\section{Group comparisons of cognitive functions in PD patients with different PWMH or DWMH gradings}

There were significant differences in MMSE scores ( $p=$ 0.002 and $p=0.001$ ) among the three groups with different DWMH gradings and those with different PWMH gradings. There were significant differences in attention function $(p=0.02)$ and memory $(p=0.04)$ among PD patients with different DWMH gradings (Table 2). In PD patients with different PWMH gradings (Table 3), there were statistical significances in the attention, memory, and language domains of cognition functions $(p=0.003$, 0.003 , and 0.04).

\section{Group comparisons of oxidative stress biomarkers in PD patients with different PWMH or DWMH gradings}

As shown in Fig. 1, there were significant differences in the percentages of total leucocyte early apoptosis, granulocyte APO 2.7, and total leucocyte APO 2.7 in PD patients with different DWMH gradings $(p=0.018,0.033$, $0.049)$. A significant difference was revealed in the percentage of total leucocyte early apoptosis between patients with normal and mild DWMHs $(p=0.015)$. There was a significant difference in the percentage of total leucocyte late apoptosis between patients with mild and moderate to severe DWMH damages $(p=0.022)$. Significant differences were seen in the percentages of granulocyte and total leucocyte APO 2.7 between patients with mild and moderate to severe DWMH damage ( $p=0.004$, 0.016). However, no significant difference was found in all detected oxidative stress biomarkers among PD patients with different PWMH gradings (Fig. 2). 
Table 2 Disease severity and neuropsychological assessment in PD patients with different DWMH gradings

\begin{tabular}{|c|c|c|c|c|c|c|c|}
\hline DWMH gradings & Normal $(n=24)$ & Mild $(n=80)$ & Moderate to Severe $(n=42)$ & $\mathbf{P}$ & $P_{1}$ & $P_{2}$ & $P_{3}$ \\
\hline Gender (male, female) & 11,13 & 30,50 & 23,19 & 0.185 & 0.484 & 0.610 & 0.084 \\
\hline Age (years, mean (SD)) & $55.37(12.99)$ & $63.06(9.85)$ & $69.61(6.72)$ & $0.001^{*}$ & $0.013^{*}$ & $0.001^{*}$ & $0.001 *$ \\
\hline HTN (number, percentage) & $8(33.3 \%)$ & $25(31.3 \%)$ & $21(50 \%)$ & 0.117 & 0.848 & 0.193 & $0.043^{*}$ \\
\hline DM (number, percentage) & $3(12.5 \%)$ & $10(12.5 \%)$ & $6(14.3 \%)$ & 0.959 & 1.000 & 0.840 & 0.782 \\
\hline Hypercholesterolemia (number, percentage) & $3(12.5 \%)$ & $16(20 \%)$ & $12(28.6 \%)$ & 0.286 & 0.407 & 0.137 & 0.287 \\
\hline Smoking (number, percentage) & $1(4.2 \%)$ & $3(3.8 \%)$ & 0 & 0.436 & 0.926 & 0.186 & 0.206 \\
\hline Medications (number, percentage) & $8(33.3 \%)$ & $30(37.5 \%)$ & $14(33.3 \%)$ & 0.873 & 0.711 & 1.000 & 0.650 \\
\hline UPDRS total, mean (SD) & $33.91(23.26)$ & $39.78(23.05)$ & $38.71(29.19)$ & 0.458 & 0.226 & 0.646 & 0.478 \\
\hline Modified Hoehn and Yahr scale, mean (SD) & $1.71(0.85)$ & $1.92(1.09)$ & $2.09(1.31)$ & 0.706 & 0.674 & 0.404 & 0.583 \\
\hline Schwab and England ADL scale mean (SD) & $87.36(12.40)$ & $82.89(16.55)$ & $81.00(22.16)$ & 0.583 & 0.281 & 0.528 & 0.741 \\
\hline MMSE, mean (SD) & $25.47(3.31)$ & $25.32(4.53)$ & $22.14(5.71)$ & $0.002^{*}$ & 0.471 & $0.021^{*}$ & $0.001^{*}$ \\
\hline \multicolumn{8}{|l|}{ Neuropsychological battery } \\
\hline Attention, mean (SD) & $-0.24(1.95)$ & $-0.20(2.03)$ & $-1.57(2.91)$ & $0.020^{*}$ & 0.899 & 0.060 & $0.006^{*}$ \\
\hline Executive function, mean (SD) & $-1.31(4.48)$ & $-0.60(4.43)$ & $-2.04(4.69)$ & 0.338 & 0.441 & 0.758 & 0.149 \\
\hline Memory, mean (SD) & $0.09(1.96)$ & $-0.34(2.17)$ & $-1.36(2.77)$ & $0.040^{*}$ & 0.620 & $0.028^{*}$ & $0.027^{*}$ \\
\hline Language, mean (SD) & $-0.54(1.73)$ & $-0.26(2.43)$ & $-1.30(2.87)$ & 0.127 & 0.431 & 0.298 & 0.051 \\
\hline Visual-spatial, mean (SD) & $-1.29(1.79)$ & $-0.56(2.12)$ & $-1.26(2.67)$ & 0.148 & 0.087 & 0.714 & 0.159 \\
\hline
\end{tabular}

Kruskal-Wallis $\mathrm{H}$ test was used for between-group comparisons of the three groups, and Mann-Whitney $\mathrm{U}$ test was used for between-two-group comparisons $P$ Comparison of group 0,1 , and $2+3, P 1$ Comparison of group 0 and $1, P 2$ Comparison of group 0 and $2+3, P 3$ Comparison of group 1 and $2+3$, SD Standard deviation, PD Parkinson's disease, DWMH Deep white matter hyperintensity, HTN Hypertension, DM Diabetes Mellitus; Medications, was defined as Benzodiazepines ${ }^{*} p<0.05$

Table 3 Disease severity and neuropsychological assessment in PD patients with different PWMH gradings

\begin{tabular}{|c|c|c|c|c|c|c|c|}
\hline PWMH gradings & Normal $(n=16)$ & Mild $(n=90)$ & moderate to severe $(n=40)$ & $\mathbf{P}$ & $\mathbf{P}_{1}$ & $\mathrm{P}_{2}$ & $P_{3}$ \\
\hline Gender (male, female) & 9,7 & 37,53 & 19,21 & 0.523 & 0.285 & 0.442 & 0.704 \\
\hline Age (years, mean (SD)) & $57.81(11.49)$ & $62.58(10.67)$ & $69.50(8.60)$ & $0.001^{*}$ & 0.067 & $0.001^{*}$ & $0.001^{*}$ \\
\hline HTN (number, percentage) & $4(25 \%)$ & $31(34.4 \%)$ & $19(47.5 \%)$ & 0.211 & 0.461 & 0.125 & 0.160 \\
\hline DM (number, percentage) & $2(12.5 \%)$ & $11(12.2 \%)$ & $6(15 \%)$ & 0.909 & 0.975 & 0.811 & 0.666 \\
\hline Hypercholesterolemia (number, percentage) & $2(12.5 \%)$ & $20(22.2 \%)$ & $9(22.5 \%)$ & 0.665 & 0.379 & 0.399 & 0.972 \\
\hline Smoking (number, percentage) & 0 & $4(4.4 \%)$ & 0 & 0.281 & 0.392 & 1.000 & 0.177 \\
\hline Medications (number, percentage) & $11(68.8 \%)$ & $28(31.1 \%)$ & $13(32.5 \%)$ & $0.014^{*}$ & $0.004^{*}$ & $0.014^{*}$ & 0.875 \\
\hline UPDRS total, mean (SD) & $43.43(22.11)$ & $36.68(23.50)$ & $41.58(29.09)$ & 0.456 & 0.229 & 0.486 & 0.555 \\
\hline Modified Hoehn and Yahr scale, mean (SD) & $2.18(1.34)$ & $1.86(1.04)$ & $2.03(1.26)$ & 0.761 & 0.445 & 0.669 & 0.803 \\
\hline Schwab and England ADL scale, mean (SD) & $82.67(13.87)$ & $83.97(16.30)$ & $80.52(22.29)$ & 0.823 & 0.527 & 0.680 & 0.841 \\
\hline MMSE, mean (SD) & $27.62(2.24)$ & $24.71(4.54)$ & $22.56(5.73)$ & $0.001^{*}$ & $0.007^{*}$ & $0.001^{*}$ & $0.033^{*}$ \\
\hline \multicolumn{8}{|l|}{ Neuropsychological battery } \\
\hline Attention, mean (SD) & $0.83(1.16)$ & $-0.46(2.17)$ & $-1.44(2.80)$ & $0.003^{*}$ & $0.030^{*}$ & $0.001^{*}$ & $0.034^{*}$ \\
\hline Executive function, mean (SD) & $0.71(3.97)$ & $-0.97(4.23)$ & $-2.23(5.11)$ & 0.113 & 0.130 & 0.060 & 0.225 \\
\hline Memory, mean (SD) & $0.78(1.14)$ & $-0.36(2.06)$ & $-1.54(2.94)$ & $0.003^{*}$ & $0.033^{*}$ & $0.001^{*}$ & $0.037^{*}$ \\
\hline Language, mean (SD) & $0.31(2.12)$ & $-0.36(2.20)$ & $-1.48(3.00)$ & $0.040^{*}$ & 0.380 & $0.044^{*}$ & $0.028^{*}$ \\
\hline Visual-spatial, mean (SD) & $0.18(1.96)$ & $-0.83(2.10)$ & $-1.35(2.57)$ & 0.058 & 0.065 & $0.029^{*}$ & 0.210 \\
\hline
\end{tabular}

Kruskal-Wallis $\mathrm{H}$ test was used for between-group comparisons of three groups, and Mann-Whitney $\mathrm{U}$ test was used for between-two-group comparisons $P$ Comparison of group 0,1 and $2+3, P 1$ Comparison of group 0 and $1, P 2$ Comparison of group 0 and $2+3, P 3$ Comparison of group 1 and $2+3$, SD Standard deviation, PD Parkinson's disease, PWMH Periventricular white matter hyperintensities, HTN Hypertension, DM Diabetes Mellitus; Medications, was defined as Benzodiazepines ${ }^{*} p<0.05$ 


\section{Leukocyte apoptosis between groups of DWMH}

Early apoptosis (\%)

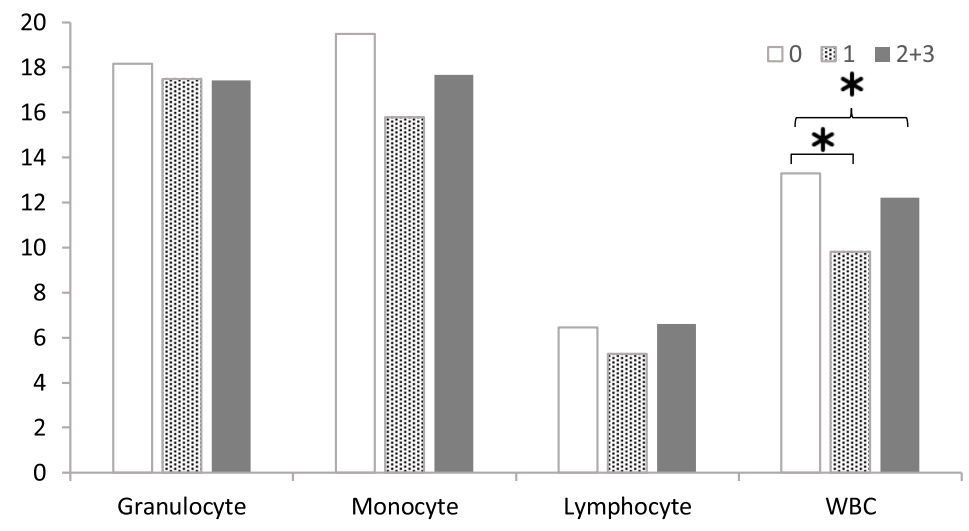

Late apoptosis (\%)

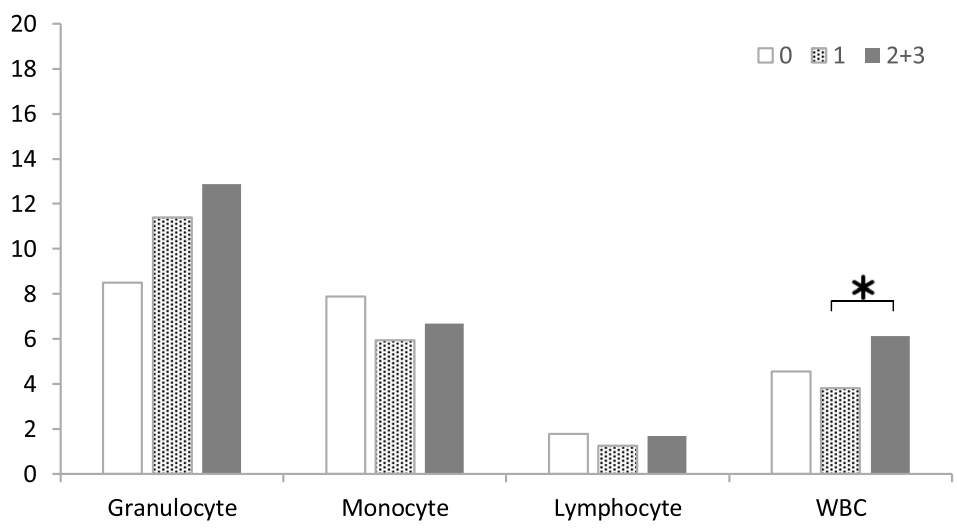

APO 2.7 apoptosis (\%)

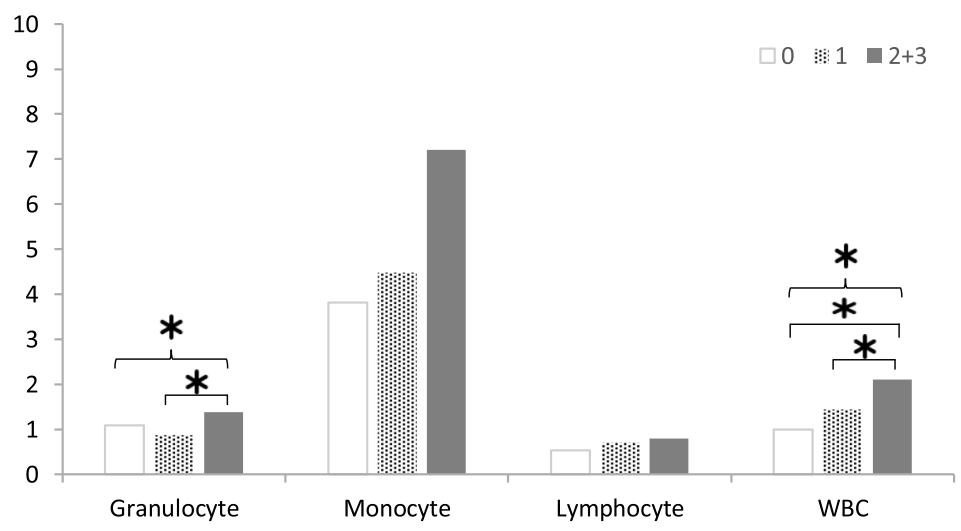

Fig. 1 Leukocyte apoptosis between groups of DWMH. The percentages of (a) early apoptosis, (b) late apoptosis, and (c) APO 2.7 apoptosis in patients with normal, mild, and moderate to severe deep white matter (DWM) lesions. Apoptotic cells were defined as APO 2.7-positive. Leucocyte subtypes were identified according to their CD45 expression intensity. The presence of late apoptotic cells meant that the cell membrane integrity was disrupted. The presence of early apoptotic cells indicated that the apoptotic change was early and still reversible. Early apoptosis of total leucocytes was lower in patients with advanced DWM lesions. However, the late apoptosis of total leucocytes and APO 2.7 apoptosis of granulocytes and total leucocytes were significantly higher in patients with advanced DWM lesions compared with normal lesions. Data are presented as mean \pm standard deviation. ${ }^{*} P<0.05$ in group comparisons. DMWH, deep white matter hyperintensities 


\section{Leukocyte apoptosis between groups of PWMH}

Early apoptosis (\%)

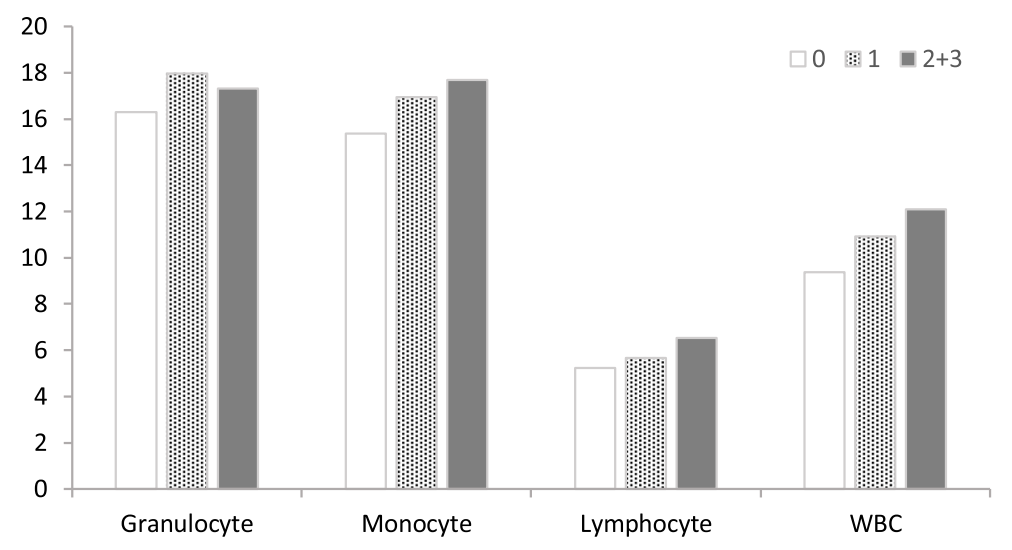

Late apoptosis (\%)

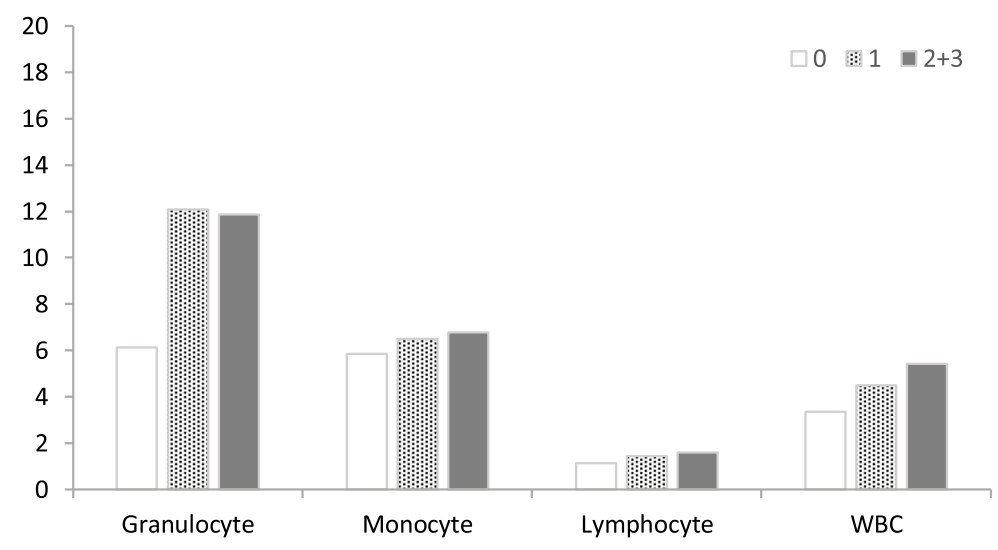

APO 2.7 apoptosis (\%)

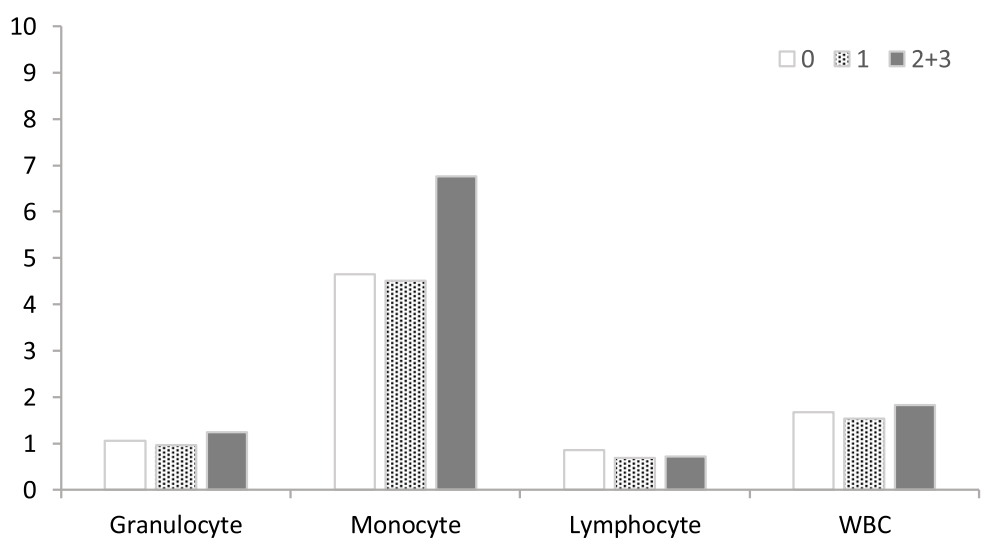

Fig. 2 Leukocyte apoptosis between groups of PWMH. The percentages of (a) early apoptosis, (b) late apoptosis, and (c) APO 2.7 apoptosis in patients with normal, mild, and moderate to severe periventricular white matter (PWM) lesions. There was no significant difference in group comparisons of normal and different gradings of PWM lesions. PMWH, periventricular white matter hyperintensities 


\section{Discussion}

We investigated the oxidative stress biomarkers and cognitive functions in patients with PD with PWMH or DWMH lesions and identified the changes in oxidative stress biomarkers and cognitive deficits between PD patients with different PWMH or DWMH gradings. According to the results, the percentages of granulocyte and total leucocyte APO 2.7 were significantly higher in patients with PD with more severe DWMH. In addition, the percentages of early apoptosis of total leucocytes were also significantly lower in patients with DWMH lesions than in those without DWMH lesions. Regarding cognitive performance, there were significant deficits in domains of cognitive function in patients with PD having more severe PWMH lesions. To the best of our knowledge, this is the first study to report the changes in the circulating oxidative biomarkers in PD patients with different PWM or DWM alternations.

Small vessel disease is common, causing substantial cognitive and physical disabilities in older peoples [41, 42]. One of its main imaging features is white matter hyperintensities [43]. The possible associated vascular risk factors include hypertension, diabetes mellitus, hypercholesterolemia and smoking [44-46], though the clear mechanisms are largely unknown. Furthermore, periventricular and deep white matter injuries were reported to be associated with different cardiovascular factors and may have different aetiologies in the elderly. Studies have proposed that DWMH is associated with higher body mass index but not arterial pressure, while PWMH may be linked to higher arterial pressure but not with BMI $[26,29]$. For the pathogenesis of white matter changes with ageing, some studies hypothesised more hypoxic/ischaemic damage in DWMHs, but greater inflammatory/metabolic components in PWMH $[39,47]$. For PD with white matter alternations, we investigated the changes in oxidative stress biomarkers and found higher percentages of granulocyte APO 2.7 and total leucocyte APO 2.7 in patients with moderate to severe DWMH damage. However, higher percentages of early apoptosis of total leucocytes was found in patients without DWMH damage in this study, and we considered it might be those PD patients without DWMH already experienced the early apoptosis before brain deep white matter structural changes detected by brain imaging. The real pathophysiology was still unknown, and it requires further study to explore. There were no significantly higher oxidative stress biomarkers in patients with PD having more severe PWM lesions. The findings for oxidative biomarkers in PD with different white matter injuries were not similar to those in previous reports on elderly individuals with DWMH or PWMH. The reason could be related to the presence of hypoxia in $\mathrm{PD}$, which related to respiratory dysfunction, including restrictive changes, upper airway obstruction, abnormal ventilatory drive, and response to medications [48], contributed by deep white matter damage and associated with increasing circulating inflammatory markers in PD. This finding could be similar to that of hypoxia in patients with severe obstructive sleep apnoea who had impaired white matter integrity due to increased systemic inflammation [22].

In the elderly, worse cognition domains, especially in memory and executive function/processing speed, were associated with PWMH rather than DWMH lesions [30]. PWMH lesions interfere with long connections, leading to negative impacts on mostly cognitive domains. Deep white matter damage is responsible for short connections that are less associated with cognitive performance but may play an important role in motor dysfunction [26]. For patients with PD, we also found that PD with more PWMH lesions had significant declines in more cognitive domains. Furthermore, we found that PD patients with more severe PWM damage had a tendency for deficits in executive function and a significant decrease in visual-spatial function compared with PD patients without PWMH lesions. The explanation for this finding is that executive and visual-spatial functions are a more complex process involving more brain areas [49], and therefore, severe PWM injuries may disrupt more long connections, leading to an obvious deficit. Additionally, PD patients with more severe deep white matter injuries also had reduced attention and memory functions in our study. Further studies are needed to identify supporting brain regions of white matter associated with cognitive functions in patients with PD.

The limitations of this study are as follows. First, all PD patients with white matter lesions were recruited from one medical centre. Second, no normal individuals were included in the control group for excluding white matter alternations due to ageing process. Third, most PD patients with less disease severity were enrolled in this study, and therefore could not represent the general population of PD. Fourth, there is a borderline significant difference in hypertension between mild and moderate to severe gradings of DWMH lesions. Further correlation between hypertension, oxidative stress and cognitive performance were not conducted in our study. In the future, we hope to conduct more studies to enrol early PD patients without white matter injury and to conduct a longitudinal study to observe their neurostructural and cognitive changes and to determine the role of neuroinflammation in $\mathrm{PD}$ with white matter injuries.

\section{Conclusion}

In summary, we found significant alternations in some oxidative stress biomarkers only in PD patients with 
DWMH, but not PWMH. Systemic inflammatory responses may contribute to deep white matter damage in $\mathrm{PD}$. In patients with $\mathrm{PD}$, more severe periventricular white matter injury was associated with more cognitive deficits, some of which were also found in patients with worse deep white matter lesions. Clinically, this study suggests practical interventions in PD to inhibit the inflammatory response and prevent or impede the progression of white matter injury related to cognitive impairments.

\section{Abbreviations}

CASI: Cognitive Ability Screening Instrument; DWMH: Deep white matter hyperintensities; HYSS: Hoehn and Yahr Staging Scale; MMSE: Mini-Mental State Examination; MRI: Magnetic resonance imaging; PD: Parkinson's disease; PWMH: Periventricular white matter hyperintensities

\section{Acknowledgements}

We thank our colleagues from the hospital who provided expertise that assisted this study.

\section{Authors' contributions}

TCC acquisition, analysis, or interpretation of data drafting of the manuscript. YCC acquisition, analysis, or interpretation of data drafting of the manuscript. YCH concept and design acquisition, analysis, or interpretation of data drafting of the manuscript critical revision of the manuscript for important intellectual content administrative, technical, or material support, supervision. WCL concept and design drafting of the manuscript critical revision of the manuscript for important intellectual content obtained funding administrative, technical, or material support, supervision. $\mathrm{CHL}$ critical revision of the manuscript for important intellectual content administrative, technical or material support. The authors read and approved the final manuscript.

\section{Funding}

This study was funded by NMRPG8J6022 (MOST 108-2314-B-182A-014-MY3), NMRPG8J0271 (MOST 108-2314-B-182A-017), and CMRPG8K0221. The authors declare that they have no competing interests.

\section{Availability of data and materials}

The datasets generated and analysed during the current study are not publicly available due to the ethical reasons but are available from the corresponding author on reasonable request.

\section{Ethics approval and consent to participate}

The Chang Gung Medical Foundation Institutional Review Board approved this retrospective study (202001300B0) with the permission of using the raw data from previous three trials (IRB 99-3612A3, 99-2833A3, and 100-4402A3) and. All of the participants or their legal guardians provided written informed consent, including this study and previous trials.

\section{Consent for publication}

The consent for publication was obtained from each participant.

\section{Competing interests}

The authors declare that they have no competing interests.

\section{Author details}

${ }^{1}$ Department of Physical Medicine and Rehabilitation, Kaohsiung Chang Gung Memorial Hospital and Chang Gung University College of Medicine, Kaohsiung, Taiwan. Department of Diagnostic Radiology, Kaohsiung Chang Gung Memorial Hospital and Chang Gung University College of Medicine, Kaohsiung, Taiwan. ${ }^{3}$ Department of Neurology, Kaohsiung Chang Gung Memorial Hospital and Chang Gung University College of Medicine, Kaohsiung, Taiwan.
Received: 28 September 2020 Accepted: 26 December 2020

Published online: 11 January 2021

\section{References}

1. Beitz JM. Parkinson's disease: a review. Front Biosci (Scholar edition). 2014;6: 65-74. https://doi.org/10.2741/s415.

2. Hornykiewicz $\mathrm{O}$. The discovery of dopamine deficiency in the parkinsonian brain. In: Parkinson's Disease and Related Disorders. Springer; 2006. p. 9-15.

3. Gerfen CR. Molecular effects of dopamine on striatal-projection pathways. Trends Neurosci. 2000;23(10 Suppl):S64-70. https://doi.org/10.1016/s14711931(00)00019-7.

4. Ekman U, Eriksson J, Forsgren L, Mo SJ, Riklund K, Nyberg L. Functional brain activity and presynaptic dopamine uptake in patients with Parkinson's disease and mild cognitive impairment: a cross-sectional study. Lancet Neurol. 2012:11(8):679-87. https://doi.org/10.1016/s1474-4422(12)70138-2.

5. Allam MF, Del Castillo AS, Navajas RF. Parkinson's disease risk factors: genetic, environmental, or both? Neurol Res. 2005;27(2):206-8. https://doi. org/10.1179/016164105×22057

6. Maries E, Dass B, Collier TJ, Kordower JH, Steece-Collier K. The role of alphasynuclein in Parkinson's disease: insights from animal models. Nat Rev Neurosci. 2003;4(9):727-38. https://doi.org/10.1038/nrn1199.

7. Su X, Federoff HJ. Immune responses in Parkinson's disease: interplay between central and peripheral immune systems. Biomed Res Int. 2014; 2014:275178. https://doi.org/10.1155/2014/275178

8. Schapira AH, Cooper JM, Dexter D, Jenner P, Clark JB, Marsden CD. Mitochondrial complex I deficiency in Parkinson's disease. Lancet (London England). 1989;1(8649):1269. https://doi.org/10.1016/s0140-6736(89)92366-0.

9. Ferrari CC, Tarelli R. Parkinson's disease and systemic inflammation. Parkinson's Dis. 2011;2011:436813. https://doi.org/10.4061/2011/436813.

10. Hald A, Lotharius J. Oxidative stress and inflammation in Parkinson's disease: is there a causal link? Exp Neurol. 2005;193(2):279-90. https://doi.org/10. 1016/j.expneurol.2005.01.013

11. Chiang PL, Chen HL, Lu CH, Chen YS, Chou KH, Hsu TW, et al. Interaction of systemic oxidative stress and mesial temporal network degeneration in Parkinson's disease with and without cognitive impairment. J Neuroinflammation. 2018;15(1):281. https://doi.org/10.1186/s12974-018$1317-z$

12. Lin WC, Chou KH, Lee PL, Huang YC, Tsai NW, Chen HL, et al. Brain mediators of systemic oxidative stress on perceptual impairments in Parkinson's disease. J Transl Med. 2015;13:386. https://doi.org/10.1186/ s12967-015-0749-9.

13. Chen YS, Chen MH, Lu CH, Chen PC, Chen HL, Yang $\mathrm{H}$, et al. Associations among cognitive functions, plasma DNA, and white matter integrity in patients with early-onset Parkinson's disease. Front Neurosci. 2017:11:9. https://doi.org/10.3389/fnins.2017.00009.

14. Chiang $\mathrm{PL}$, Chen $\mathrm{HL}$, Lu CH, Chen $\mathrm{PC}$, Chen MH, Yang $\mathrm{IH}$, et al. White matter damage and systemic inflammation in Parkinson's disease. BMC Neurosci. 2017:18(1):48. https://doi.org/10.1186/s12868-017-0367-y.

15. Lin WC, Tsai NW, Huang YC, Cheng KY, Chen HL, Li SH, et al. Peripheral leukocyte apoptosis in patients with parkinsonism: correlation with clinical characteristics and neuroimaging findings. Biomed Res Int. 2014;2014: 635923. https://doi.org/10.1155/2014/635923.

16. Sasaki T, Senda M. Evaluation of glutathione localization in brain using 99mTc meso-HMPAO. J Nucl Med. 1999:40(6):1056-60.

17. Wardlaw JM, Smith EE, Biessels GJ, Cordonnier C, Fazekas F, Frayne R, et al. Neuroimaging standards for research into small vessel disease and its contribution to ageing and neurodegeneration. Lancet Neurol. 2013;12(8): 822-38

18. Longstreth WT Jr, Manolio TA, Arnold A, Burke GL, Bryan N, Jungreis CA et al. Clinical correlates of white matter findings on cranial magnetic resonance imaging of 3301 elderly people. The Cardiovascular Health Study. Stroke. 1996;27(8):1274-82. https://doi.org/10.1161/01.str.27.8.1274.

19. Alber J, Alladi S, Bae HJ, Barton DA, Beckett LA, Bell JM, et al. White matter hyperintensities in vascular contributions to cognitive impairment and dementia (VCID): Knowledge gaps and opportunities. Alzheimer's Dementia (New York, N Y). 2019:5:107-17. https://doi.org/10.1016/j.trci.2019.02.001.

20. Makino T, Umegaki H, Suzuki Y, Yanagawa M, Nonogaki Z, Nakashima H, et al. Relationship between small cerebral white matter lesions and cognitive function in patients with Alzheimer's disease and amnestic mild cognitive impairment. Geriatr Gerontol Int. 2014;14(4):819-26. https://doi. org/10.1111/ggi.12176. 
21. Huang X, Wen MC, Ng SY, Hartono S, Chia NS, Choi X, et al. Periventricular white matter hyperintensity burden and cognitive impairment in early Parkinson's disease. Eur J Neurol. 2020;27(6):959-66. https://doi.org/10.1111/ ene.14192.

22. Chen HL, Lu CH, Lin HC, Chen PC, Chou KH, Lin WM, et al. White matter damage and systemic inflammation in obstructive sleep apnea. Sleep. 2015; 38(3):361-70. https://doi.org/10.5665/sleep.4490.

23. Stolp HB, Dziegielewska KM. Review: role of developmental inflammation and blood-brain barrier dysfunction in neurodevelopmental and neurodegenerative diseases. Neuropathol Appl Neurobiol. 2009;35(2):13246. https://doi.org/10.1111/j.1365-2990.2008.01005.x.

24. Kandiah N, Mak E, Ng A, Huang S, Au WL, Sitoh YY, et al. Cerebral white matter hyperintensity in Parkinson's disease: a major risk factor for mild cognitive impairment. Parkinsonism Relat Disord. 2013;19(7):680-3. https:// doi.org/10.1016/j.parkreldis.2013.03.008.

25. Dalaker TO, Larsen JP, Dwyer MG, Aarsland D, Beyer MK, Alves G, et al. White matter hyperintensities do not impact cognitive function in patients with newly diagnosed Parkinson's disease. Neuroimage. 2009;47(4):2083-9. https://doi.org/10.1016/j.neuroimage.2009.06.020.

26. Griffanti L, Jenkinson M, Suri S, Zsoldos E, Mahmood A, Filippini N, et al. Classification and characterization of periventricular and deep white matter hyperintensities on MRI: a study in older adults. Neuroimage. 2018;170:17481.

27. Scheltens P, Barkhof F, Leys D, Pruvo JP, Nauta J, Vermersch P, et al. A semiquantative rating scale for the assessment of signal hyperintensities on magnetic resonance imaging. J Neurol Sci. 1993;114(1):7-12.

28. Debette $\mathrm{S}$, Markus $\mathrm{H}$. The clinical importance of white matter hyperintensities on brain magnetic resonance imaging: systematic review and meta-analysis. Bmj. 2010;341:c3666.

29. Kim KW, MacFall JR, Payne ME. Classification of white matter lesions on magnetic resonance imaging in elderly persons. Biol Psychiatry. 2008;64(4): 273-80

30. Bolandzadeh N, Davis JC, Tam R, Handy TC, Liu-Ambrose T. The association between cognitive function and white matter lesion location in older adults: a systematic review. BMC Neurol. 2012;12(1):126.

31. Hughes AJ, Ben-Shlomo Y, Daniel SE, Lees AJ. What features improve the accuracy of clinical diagnosis in Parkinson's disease: a clinicopathologic study. Neurology. 1992;42(6):1142.

32. Ramaker C, Marinus J, Stiggelbout AM, Van Hilten BJ. Systematic evaluation of rating scales for impairment and disability in Parkinson's disease. Mov Disord. 2002;17(5):867-76.

33. Goetz CG, Poewe W, Rascol O, Sampaio C, Stebbins GT, Counsell C, et al. Movement Disorder Society task force report on the Hoehn and Yahr staging scale: status and recommendations the Movement Disorder Society task force on rating scales for Parkinson's disease. Mov Disord. 2004;19(9): 1020-8.

34. Schwab RS. Projection technique for evaluating surgery in Parkinson's disease. In: Third symposium on Parkinson's disease. E\&S Livingstone; 1969. p. 152-7.

35. Teng EL, Hasegawa K, Homma A, Imai Y, Larson E, Graves A, et al. The cognitive abilities screening instrument (CASI): a practical test for crosscultural epidemiological studies of dementia. Int Psychogeriatr. 1994;6(1):4558; discussion 62. https://doi.org/10.1017/s1041610294001602.

36. Taylor MJ, Heaton RK. Sensitivity and specificity of WAIS-III/WMS-III demographically corrected factor scores in neuropsychological assessment. J Int Neuropsychol Soc. 2001;7(7):867-74.

37. Lin WC, Huang YC, Leong CP, Chen MH, Chen HL, Tsai NW, et al. Associations between cognitive functions and physical frailty in patients with Parkinson's disease. Front Aging Neurosci. 2019;11:283. https://doi.org/ 10.3389/fnagi.2019.00283.

38. Fazekas F, Chawluk JB, Alavi A, Hurtig HI, Zimmerman RA. MR signal abnormalities at $1.5 \mathrm{~T}$ in Alzheimer's dementia and normal aging. AJR Am J Roentgenol. 1987;149(2):351-6. https://doi.org/10.2214/ajr.149.2.351.

39. Fazekas F, Kleinert $\mathrm{R}$, Offenbacher $\mathrm{H}$, Schmidt $\mathrm{R}$, Kleinert $\mathrm{G}$, Payer $\mathrm{F}$, et al. Pathologic correlates of incidental MRI white matter signal hyperintensities. Neurology. 1993;43(9):1683-9. https://doi.org/10.1212/wnl.43.9.1683.

40. van den Heuvel DM, ten Dam VH, de Craen AJ, Admiraal-Behloul F, Olofsen $\mathrm{H}$, Bollen EL, et al. Increase in periventricular white matter hyperintensities parallels decline in mental processing speed in a non-demented elderly population. J Neurol Neurosurg Psychiatry. 2006;77(2):149-53. https://doi. org/10.1136/jnnp.2005.070193.
41. van der Flier WM, van Straaten EC, Barkhof F, Verdelho A, Madureira S,

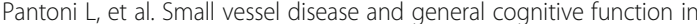
nondisabled elderly: the LADIS study. Stroke. 2005;36(10):2116-20. https:// doi.org/10.1161/01.Str.0000179092.59909.42.

42. de Laat KF, Tuladhar AM, van Norden AG, Norris DG, Zwiers MP, de Leeuw FE. Loss of white matter integrity is associated with gait disorders in cerebral small vessel disease. Brain. 2011;134(Pt 1):73-83. https://doi.org/10. 1093/brain/awq343.

43. Rost NS, Rahman RM, Biffi A, Smith EE, Kanakis A, Fitzpatrick K, et al. White matter hyperintensity volume is increased in small vessel stroke subtypes. Neurology. 2010;75(19):1670-7. https://doi.org/10.1212/WNL. 0b013e3181fc279a.

44. Dufouil C, de Kersaint-Gilly A, Besançon V, Levy C, Auffray E, Brunnereau L, et al. Longitudinal study of blood pressure and white matter hyperintensities: the EVA MRI cohort. Neurology. 2001;56(7):921-6. https:// doi.org/10.1212/wnl.56.7.921.

45. van Dijk EJ, Prins ND, Vrooman HA, Hofman A, Koudstaal PJ, Breteler MM. Progression of cerebral small vessel disease in relation to risk factors and cognitive consequences: Rotterdam scan study. Stroke. 2008;39(10):2712-9. https://doi.org/10.1161/strokeaha.107.513176.

46. Jackson CA, Hutchison A, Dennis MS, Wardlaw JM, Lindgren A, Norrving B, et al. Differing risk factor profiles of ischemic stroke subtypes: evidence for a distinct lacunar arteriopathy? Stroke. 2010;41(4):624-9. https://doi.org/10. 1161/strokeaha.109.558809.

47. Fernando MS, Simpson JE, Matthews F, Brayne C, Lewis CE, Barber R, et al. White matter lesions in an unselected cohort of the elderly: molecular pathology suggests origin from chronic hypoperfusion injury. Stroke. 2006; 37(6):1391-8. https://doi.org/10.1161/01.Str.0000221308.94473.14.

48. Baille G, De Jesus AM, Perez T, Devos D, Dujardin K, Charley CM, et al. Ventilatory dysfunction in Parkinson's disease. J Parkinsons Dis. 2016;6(3): 463-71. https://doi.org/10.3233/jpd-160804.

49. Bagarinao E, Watanabe H, Maesawa S, Mori D, Hara K, Kawabata K, et al. Reorganization of brain networks and its association with general cognitive performance over the adult lifespan. Sci Rep. 2019;9(1):11352. https://doi. org/10.1038/s41598-019-47922-x.

\section{Publisher's Note}

Springer Nature remains neutral with regard to jurisdictional claims in published maps and institutional affiliations.

Ready to submit your research? Choose BMC and benefit from

- fast, convenient online submission

- thorough peer review by experienced researchers in your field

- rapid publication on acceptance

- support for research data, including large and complex data types

- gold Open Access which fosters wider collaboration and increased citations

- maximum visibility for your research: over $100 \mathrm{M}$ website views per year

At $\mathrm{BMC}$, research is always in progress.

Learn more biomedcentral.com/submission 